\title{
Herausforderungen und Chancen von Startups bei der Erstfinanzierung
}

\author{
Der Artikel beschreibt Herausforderungen und Chancen von Startups bei der \\ Suche nach einem ersten Kapitalgeber. Anschließend werden Chancen zur \\ Bewältigung der Herausforderungen eröffnet.
}
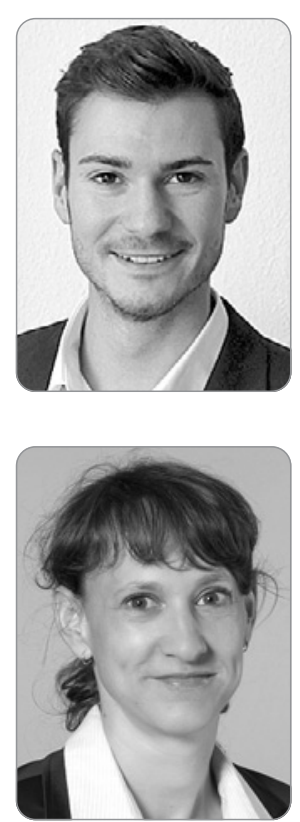

Bastian Lontzek, B.Eng.,

war Student des Wirtschaftsingenieurwesens an der Hochschule für angewandte Wissenschaften München. Sein Schwerpunkt legte er auf die Gründung und Finanzierung von Unternehmen und arbeitete für seine Abschlussarbeit eng mit einem Startup des Strascheg Center for Entrepreneurship (SCE) zusammen. Bevorzugtes Forschungsgebiet: Unternehmensfinanzierung und -gründung.

\section{Prof. Dr. Verena McIntosh}

ist Professorin für Finanzierung, Bilanzierung und Betriebswirtschaftslehre an der Hochschule für angewandte Wissenschaften München. Bevorzugte Forschungsgebiete: Kapitalmärkte und Financial Risk Management.

Stichwörter: Startup; Finanzierung; Herausforderung; Chance; Kapitalgeber

\section{Wirtschaftliche Bedeutung von Startups}

Startups nehmen eine wichtige volkswirtschaftliche Rolle ein: Sie sind Treiber für den inländischen sowie internationalen Wettbewerb, sie schaffen neue Arbeitsplätze und stärken die Wirtschaft durch neue Innovationen (vgl. Bundesministerium für Wirtschaft und Energie, 2016).

\section{Herausforderungen und Chancen von Startups bei der Erstfinanzierung}

Um ein Gründungsvorhaben umsetzen zu können, werden in der Regel finanzielle Mittel benötigt. Laut einem Bericht der KfW Bankengruppe scheitert jedoch rund die Hälfte der Gründungsvorhaben an einer erfolgreichen Erstfinanzierung (vgl. Metzger, 2015, S. 4). Denn in der Praxis stehen junge Unternehmen einer Vielzahl von finanziellen Heraus- forderungen gegenüber. Einige typische Schwierigkeiten von Startups bei der ersten Finanzierungsrunde werden im Folgenden erörtert. Die Analyse wurde mit einer Unternehmensbefragung fundiert (vgl. Lontzek, 2017, S. 39 ff.).

\subsection{Stellenwert des Umsatzes als Investitionskriterium}

Zum ersten stehen junge Unternehmen vor der Herausforderung, einen Kapitalgeber zu gewinnen, noch bevor erste Umsätze generiert wurden. Knapp die Hälfte der befragten Startups, die eine Absage erhielten, erhielten diese aufgrund noch nicht vorhandener Umsätze. Denn um das Verlustrisiko zu evaluieren, analysieren Kapitalgeber die Finanz-Kennzahlen der Startups. Dabei stehen insbesondere die Umsatz- und Rentabilitätsgrößen im Fokus (vgl. Grummer/Brorhilker 2013), die bei Startups in der Anlaufzeit negativ ausfallen.

Der Umkehrschluss, ohne Umsatz keine Investoren zu kontaktieren, da dies sinnlos sei, hielt der empirischen Überprüfung gleichwohl nicht stand. Mehr als drei Viertel der befragten Startups absolvierten die erste Finanzierungsrunde erfolgreich innerhalb der frühen Early Stage (PreSeed und Seed-Phase), obwohl in diesen Phasen in der Regel noch keine Erlöse generiert werden (vgl. Kollmann, 2016, S. 123). So sind durchaus Investoren bereit, das hohe Ausfallrisiko auf sich zu nehmen. Besonders Business Angels spielen hier eine große Rolle. Knapp zwei Drittel der jungen Unternehmen wurden durch eine Privatperson finanziert.

Auch Venture Capital kann in der Frühphasenfinanzierung zum Erfolg führen. Etwa jedes fünfte der befragten Startups konnte einen Venture Capital Geber für die erste Finanzierungsrunde gewinnen. Ein ähnliches, noch etwas positiveres, Bild zeigt die Statistik des Bundesverbandes Deutscher Kapitalbeteiligungs-Gesellschaften aus dem Jahr 2016: Mehr als ein Viertel der Risikokapitalbeteiligungen wurden mit Unternehmen der Seed-Phase eingegangen (vgl. Bundesverband Deutscher Kapitalbeteiligungs-Gesellschaften, 2017). 
Abb. 1: Beispiel eines Ablaufes des gesamten Finanzierungsprozesses

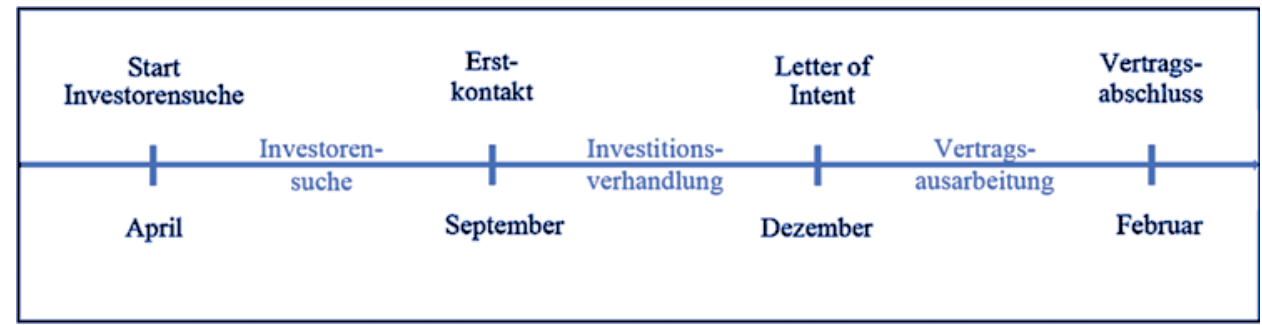

\subsection{Zeit als Hürde und Chance des Finanzierungsprozesses}

Bis jedoch eine Finanzierung final abgeschlossen ist, ist es - unabhängig von der Finanzierungsquelle - ein langer Prozess (vgl. Abb. 1). Zunächst muss ein potenzieller Kapitalgeber gefunden und von der eigenen Geschäftsidee überzeugt werden. Dieser Prozess der Investorensuche erstreckt sich über ungefähr ein halbes Jahr. Der anschließende Verhandlungsprozess mit dem Kapitalgeber nimmt ebenfalls im Schnitt sechs Monate in Anspruch. Dabei lassen sich Unterschiede zwischen den verschiedenen Finanzierungsquellen beobachten. Mit rund zehn Monaten dauern die Verhandlungen mit Venture Capital Gesellschaften am längsten. Durchschnittlich ein halbes Jahr dauern die Gespräche mit Business Angels, staatlichen Förderinstitutionen und strategischen Investoren. Mit einem Zeitraum von ein bis zwei Monaten geht die Kapitalaufnahme über die Bank am schnellsten (vgl. Lontzek, 2017, S. 39 ff.).

Vor dem Hintergrund dieses zeitintensiven Prozesses stehen die Gründer vor der Herausforderung, die Überbrückungs-Finanzierung zu stemmen. Die hierfür benötigten finanziellen Mittel werden schwerpunktmäßig aus persönlichem Vermögen entnommen oder stammen von der Familie und/oder Freunden. Da diese Reserven häufig nur für einen kurzen Zeitraum reichen, ist eine frühe externe Kapitalaufnahme zentral. Es empfiehlt sich, mindestens sechs bis acht Monate, bevor die Planung einen Liquiditätsengpass aufzeigen würde, mit der Suche nach einem Kapitalgeber zu beginnen. Dies insbesondere, um Verhandlungen unter Druck zu vermeiden und einen Abschluss mit zu niedriger Bewertung oder nachteiliger Vertragsgestaltung zu riskieren (vgl. Graber, 2016).

Neben der Minimierung des Insolvenzrisikos hat die frühe und intensive Auseinandersetzung mit den Unternehmenszahlen einen weiteren Vorteil: Sie schafft Klarheit über das eigene Geschäftsmodell, identifiziert potenzielle Schwachstellen und trägt somit zu einer Weiterentwicklung des Unternehmens bei (vgl. Hoisl, 2013).

\subsection{Finden und Auswahl eines geeigneten Investors}

Eine zweite große Hürde für Startups ist, einen zum eigenen Unternehmen passenden Kapitalgeber zu finden. Für ein Viertel der befragten Startups war der sog. Startup-Investor-Fit die größte Herausforderung bei dessen Erstfinanzierung. Denn neben der Bereitstellung von Kapital sind die nicht-finanziellen Leistungen wie Branchenerfahrung oder unternehmerisches Know-How wertvoll für Unternehmer.

Für einen Startup-Investor-Fit muss im ersten Schritt zunächst das geeignete Finanzierungsinstrument ermittelt werden. Dieses ergibt sich aus den drei Kriterien:

- Umfang der Finanzierung

- Unternehmensphase des Startups

- Commitment (Mitwirken und Unterstützung) des Kapitalgebers,

Steht das angestrebte Finanzierungsinstrument fest, beginnt die eigentliche Suche nach dem "richtigen“ Investor (vgl. Hofmann, 2012).

Für die Auswahl des Kapitalgebers werden investor-spezifische Eigenschaften analysiert. Neben öffentlich verfügbaren Informationen liefert das Unternehmensportfolio des Investors wichtige Rückschlüsse auf die Kompatibilität mit dem eigenen Unternehmen (vgl. Kaczmarek, 2013). Ein kompetenter Kapitalgeber sollte über ein durchdachtes Portfolio aus gut zusammenpassenden Startups verfügen. Dabei wird ersichtlich, welche Strategie verfolgt wird, z. B. ein Fokus auf Unternehmen mit innovativen Technologien oder auf Unternehmen mit skalierbaren Geschäftsmodellen. Lässt sich eine klare Strategie erkennen und können im 0ptimalfall bereits erfolgreiche Exits aufgewiesen werden, steigt das Interesse an diesem Kapitalgeber (vgl. von Hassell, 2015). Eine sehr pragmatische Analysemöglichkeit ist es, die Portfoliounternehmen des Kapitalgebers direkt zu kontaktieren und sich Informationen über den Investor einzuholen. "Ein guter Investor hat damit keine Probleme“, sagt Business Angel Roland Zeller (Heer, 2014). Mit dieser Vorgehensweise lässt sich etwa die Arbeitsweise oder das Krisenverhalten bewerten. Zu guter Letzt ist als zentraler Faktor die zwischenmenschliche Beziehung zwischen dem Gründer und dem Kapitalgeber zu erwähnen. Da ein Startup in der Regel mehrere Jahre mit einem Investor zusammenarbeitet, spielt Sympathie eine wichtige Rolle. Am besten lässt sich diese durch persönliche Gespräche eruieren (vgl. Kaczmarek, 2013). 


\section{Bezugspreise 2020}

\section{WiSt - Wirtschaftswissenschaftliches Studium}

Inkl. WiSt Archiv online für einen Arbeitsplatz

In Gemeinschaft mit dem Verlag Franz Vahlen.

Die Bezugspreise (einschließlich MwSt.)

betragen ab 1. Januar 2020:

\section{Normalpreis}

halbjährlich €117,50

Vorzugspreis für Studenten fachbezogener

Studiengänge (gegen Nachweis) halbjährlich € 57,50

Kombipreis inkl. Campuslizenz halbjährlich €179,50

jeweils zuzüglich Vertriebsgebühren

halbjährlich $€ 8,10$.

Abbestellung bis 6 Wochen vor Halbjahresende.

Einzelheft

Bestellen Sie bei Ihrem Buchhändler oder bei:

beck-shop.de oder Verlag C.H.BECK · 80791 München · Fax: 089/38189-358 · www.beck.de

\section{(B) \\ $\overline{\text { C.H.BECK }}$}

\section{Unsere Mediaberatung}

\section{für Insertionsfragen}

\section{Stellenmarkt Kanzleien \\ A-K \\ Daniela Uphoff \\ Telefon (089) 38189-610 \\ daniela.uphoff@beck.de \\ $\underline{\mathrm{L}-\mathrm{Z}}$ \\ Christina Stauber \\ Telefon (089) 38189-681 \\ christina.stauber@beck.de}

\section{Stellenmarkt Unternehmen}

Julie von Steuben

Telefon (089) 38189-608

julie.steuben@beck.de

Aus- und Weiterbildung I

Lehre + Forschung

Daniela Uphoff

Telefon (089) 38189-610

daniela.uphoff@beck.de
Verlage I Verbände I Öffentliche Einrichtungen

Andrea Horn

Telefon (089) 38189-607

andrea.horn@beck.de

\section{IT I Software}

Christina Stauber

Telefon (089) 381 89-681

christina.stauber@beck.de

\section{Keykunden}

(Kanzleien I Aus- und Weiter-

bildung I Lehre + Forschung)

Thomas Hepp

Anzeigenverkaufsleiter

Telefon (089) 38189-612

thomas.hepp@beck.de

Allgemeine Anfragen

Telefon (089) 38189-687

Telefax (089) 3 81 89-589

mediaberatung@beck.de

\section{Verlag C.H.BECK oHG}

Wilhelmstraße 9, 80801 München

Postfach 40 0340, 80703 München

Fax (089) 38189-589

\section{Literatur}

Bundesministerium für Wirtschaft und Energie, Existenzgründung - Motor für Wachstum und Wettbewerb, 2016, Online im Internet: URL: https:// www.bmwi.de/Redaktion/DE/Dossier/existenzgruendung.html (Abrufdatum: 14.03.2017).

Bundesverband Deutscher Kapitalbeteiligungsgesellschaften (Hrsg.), BVKStatistik 2016, Berlin 2017.

Graber, F., Startup sucht Investor: Die Hürden auf dem Weg zu frischem Geld, 2016, Online im Internet: URL: https://www.derbrutkasten.com/a/ startup-sucht-investor-frisches-geld-huerden/?xtor=CS1-15 (Abrufdatum: 27.03.2017).

Grummer J.-M., Brorhilker, J., So wählen Investoren ihre Investments, 2013, Online im Internet: URL: http://www.gruenderszene.de/finanzen/i nvestoren-beteiligungswurdigkeit (Abrufdatum: 19.03.2017).

von Hassell, J., Wie Start-ups einen guten Venture Capital Investor finden, 2015, Online im Internet: URL: https://www.deutsche-startups.de/2015/ 08/11/wie-start-ups-einen-guten-venture-capital-investor-finden/(Abrufdatum: 30.03.2017).

Heer, A., So finden Sie den richtigen Investor, 2014, Online im Internet: URL: https://magazin.swisscom.ch/allgemein/so-finden-sie-den-richtig en-investor/ (Abrufdatum: 08.04.2017).

Hofmann, A., So finden Gründer den richtigen Investor, 2012, Online im Internet: URL: http://www.gruenderszene.de/allgemein/investor-finden -venture-capital (Abrufdatum: 27.03.2017).

Hoisl, B., Finanzmodell für Startups - Vorgehensweise für die Gewinn- und Verlustrechnung, 2013, Online im Internet: URL: http://startup-stuttgart. de/finanzmodell-fur-startups-vorgehensweise-guv/(Abrufdatum: 13.03.2017).

Kaczmarek, J., Welcher Investor ist der richtige?, 2013, Online im Internet: URL: http://www.gruenderszene.de/allgemein/investor-finden (Abrufdatum: 28.03.2017).

Kollmann, T., E-Entrepreneurship: Grundlagen der Unternehmensgründung in der Digitalen Wirtschaft, 6. Aufl., Wiesbaden 2016.

Lontzek, B., Herausforderungen und Chancen von Startups bei der Erstfinanzierung, München 2017.

Metzger, G., Deutschland einig Gründerland? Mitnichten! Ein Bundesländervergleich Berlin., 2015, Online im Internet: URL: https://www.kfw.de/ KfW-Konzern/Newsroom/Aktuelles/News/News-Details_313088.html (Zugriff am: 22.03.2017).

Paßmann, T., Unternehmensbewertung in der Frühphase, München 2008. 